\title{
Correlation between lithium abundances and ages of solar twin stars ${ }^{\star}$
}

\author{
Marília Carlos $^{1}$, Poul E. Nissen ${ }^{2}$ and Jorge Meléndez ${ }^{1}$ \\ 1 Universidade de São Paulo, IAG, Departamento de Astronomia, Rua do Matão 1226, Cidade Universitária, \\ 05508-900 São Paulo, SP, Brazil \\ e-mail: marilia.carlos@usp.br \\ 2 Aarhus University, Stellar Astrophysics Centre, Department of Physics and Astronomy, Ny Munkegade 120, \\ 8000 Aarhus C, Denmark
}

Received 30 September 2015 / Accepted 18 December 2015

\section{ABSTRACT}

\begin{abstract}
Aims. We want to determine the lithium abundances of solar twin stars as a function of stellar age to provide constraints for stellar evolutions models and to investigate whether there is a connection between low Li abundance and the occurrence of planets.

Methods. For a sample of 21 solar twins observed with the HARPS spectrograph at high spectral resolution $(R \simeq 115.000)$ and very high signal-to-noise ratio $(600 \leq S / N \leq 2400)$, precise lithium abundances were obtained by spectral synthesis of the Li I $6707.8 \AA$ line and compared to stellar ages, masses, and metallicities determined from a spectroscopic analysis of the same set of HARPS spectra.

Results. We show that for the large majority of the solar twins there is a strong correlation between lithium abundance and stellar age. As the age increases from 1 to $9 \mathrm{Gyr}$, the $\mathrm{Li}$ abundance decreases by a factor of $\sim 50$. The relation agrees fairly well with predictions from non-standard stellar evolution models of Li destruction at the bottom of the upper convection zone. Two stars deviate from the relation by having $\mathrm{Li}$ abundances enhanced by a factor of $\sim 10$, which may be due to planet engulfment. On the other hand, we find no indication of a link between planet hosting stars and enhanced lithium depletion.
\end{abstract}

Key words. stars: abundances - stars: evolution - planetary systems

\section{Introduction}

Lithium is destroyed in the inner layers of stars via proton capture $\left({ }^{7} \operatorname{Li}(p, \alpha) \alpha\right)$ at temperatures near to $2.5 \times 10^{6} \mathrm{~K}$. Because $\mathrm{Li}$ burning happens when the element is transported to the innermost and hotter regions through convective motions in the star, Li abundance studies offer an excellent opportunity to understand the extent of the mixing processes within and below the stellar convective zone and, therefore, are important for constraining transport mechanisms in stars.

Since the amount of lithium burning depends on the convective zone thickness, which depends on mass and metallicity, we can improve our understanding of the structure and evolution of a star by analyzing the lithium abundance dependence of these variables. It is important to note that the lithium content could also have other dependencies such as planets around a star; for instance, planetary formation could change the initial angular momentum of the star, which, according to Takeda et al. (2010) and Gonzalez et al. (2010), increases the lithium burning. Another alternative to an anomalous Li abundance is planet engulfment, which may lead to an increase in the Li abundance of a star (Montalbán \& Rebolo 2002; Sandquist et al. 2002).

Although several factors control the level of lithium burning, there is a debate regarding the lithium depletion in solar twin stars. There are two main interpretations. One is that the

* Based on data products from observations made with ESO Telescopes at the La Silla Paranal Observatory, (observing programs 072.C-0488, 183.C-0972, 188.C-0265, and 0.88.C-0323). degree of lithium depletion primarily depends on stellar age, in the sense that older stars have lower Li abundances than younger ones as has been shown by Baumann et al. (2010), Monroe et al. (2013), Meléndez et al. (2014), and Tucci Maia et al. (2015). This is supported by several models such as Andrássy \& Spruit (2015), Charbonnel \& Talon (2005), Denissenkov (2010), Do Nascimento et al. (2009), and Xiong \& Deng (2009). The second interpretation is that enhanced $\mathrm{Li}$ depletion is due to the presence of planets (Israelian et al. 2009; Sousa et al. 2010; Delgado Mena et al. 2014; Figueira et al. 2014; Gonzalez 2015) and that the degree of lithium depletion does not depend on age for stars older than 2 Gyr (Sousa et al. 2010; Delgado Mena et al. 2014).

In this paper we revisit the discussion on lithium abundance depletion in solar twin stars and its correlations with stellar age, metallicity, mass, and planet occurrence based on new, very precise $\mathrm{Li}$ abundances derived from HARPS spectra of 21 solar twins with ages between 0.7 and $8.8 \mathrm{Gyr}$.

\section{Spectra and stellar parameters}

The solar twin stars included in this paper have been applied by Nissen (2015) to study trends of abundance ratios as a function of stellar age and elemental condensation temperature. The stars were selected from Sousa et al. (2008) to have effective temperatures $\left(T_{\text {eff }}\right)$, surface gravities $(\log g)$, and metallicities $([\mathrm{Fe} / \mathrm{H}])$ close to the solar values and to have HARPS spectra (Mayor et al. 2003) with a signal-to-noise ratio $S / N \geq 600$ after 
Table 1. Li abundances, ages, masses, and stellar parameters.

\begin{tabular}{|c|c|c|c|c|c|c|c|c|}
\hline Star & $\begin{array}{c}A(\mathrm{Li}) \mathrm{LTE} \\
(\mathrm{dex})\end{array}$ & $\begin{array}{l}A(\mathrm{Li}) \text { NLTE } \\
(\mathrm{dex})\end{array}$ & $\begin{array}{l}\text { Age } \\
(\mathrm{Gyr})\end{array}$ & $\begin{array}{l}\text { Error } \\
(\mathrm{Gyr})\end{array}$ & $\begin{array}{l}\text { Mass } \\
\left(M_{\odot}\right) \\
\end{array}$ & $\begin{array}{l}T_{\text {eff }} \\
(\mathrm{K})\end{array}$ & $\begin{array}{l}\log g \\
(\operatorname{dex})\end{array}$ & $\begin{array}{c}{[\mathrm{Fe} / \mathrm{H}]} \\
(\mathrm{dex})\end{array}$ \\
\hline HD 2071 & $1.39_{-0.04}^{+0.02}$ & $1.43_{-0.04}^{+0.02}$ & 3.5 & 0.8 & 0.97 & 5724 & 4.490 & -0.084 \\
\hline HD 8406 & $1.69_{-0.01}^{+0.01}$ & $1.73_{-0.01}^{+0.01}$ & 4.2 & 0.8 & 0.96 & 5730 & 4.479 & -0.105 \\
\hline HD $20782^{a}$ & $0.67_{-0.12}^{+0.08}$ & $0.71_{-0.12}^{+0.08}$ & 7.5 & 0.4 & 0.97 & 5776 & 4.345 & -0.058 \\
\hline HD 27063 & $1.67_{-0.01}^{+0.02}$ & $1.71_{-0.01}^{+0.02}$ & 2.6 & 0.6 & 1.04 & 5779 & 4.469 & 0.064 \\
\hline HD 28471 & $0.86_{-0.09}^{+0.10}$ & $0.90_{-0.09}^{+0.10}$ & 7.0 & 0.4 & 0.97 & 5754 & 4.380 & -0.054 \\
\hline HD 38277 & $1.56_{-0.03}^{+0.03}$ & $1.58_{-0.03}^{+0.03}$ & 7.3 & 0.4 & 1.01 & 5860 & 4.270 & -0.070 \\
\hline HD $45184^{b}$ & $2.08_{-0.02}^{+0.01}$ & $2.10_{-0.02}^{+0.01}$ & 2.7 & 0.5 & 1.06 & 5871 & 4.445 & 0.047 \\
\hline HD $45289^{*}$ & $\leq 0.57$ & $\leq 0.61$ & 8.5 & 0.4 & 1.00 & 5718 & 4.284 & -0.020 \\
\hline HD 71334 & $0.58_{-0.11}^{+0.14}$ & $0.62_{-0.11}^{+0.14}$ & 8.1 & 0.4 & 0.94 & 5701 & 4.374 & -0.075 \\
\hline HD 78429 & $0.59_{-0.19}^{+0.11}$ & $0.63_{-0.19}^{+0.11}$ & 7.5 & 0.4 & 1.04 & 5756 & 4.272 & 0.078 \\
\hline HD 88084 & $0.96_{-0.06}^{+0.12}$ & $1.00_{-0.06}^{+0.12}$ & 6.0 & 0.6 & 0.96 & 5768 & 4.424 & -0.091 \\
\hline HD 92719 & $1.88_{-0.01}^{+0.01}$ & $1.90_{-0.01}^{+0.01}$ & 2.7 & 0.6 & 0.99 & 5813 & 4.488 & -0.112 \\
\hline HD 96116 & $2.19_{-0.01}^{+0.01}$ & $2.21_{-0.01}^{+0.01}$ & 0.7 & 0.7 & 1.05 & 5846 & 4.503 & 0.006 \\
\hline HD 96423 & $1.88_{-0.01}^{+0.01}$ & $1.93_{-0.01}^{+0.01}$ & 6.0 & 0.4 & 1.03 & 5714 & 4.359 & 0.113 \\
\hline HD 134664 & $2.08_{-0.01}^{+0.02}$ & $2.11_{-0.01}^{+0.02}$ & 2.3 & 0.5 & 1.07 & 5853 & 4.452 & 0.093 \\
\hline HD 146233 & $1.58_{-0.03}^{+0.02}$ & $1.62_{-0.03}^{+0.02}$ & 3.8 & 0.5 & 1.04 & 5809 & 4.434 & 0.046 \\
\hline HD 183658 & $1.24_{-0.07}^{+0.04}$ & $1.28_{-0.07}^{+0.04}$ & 5.0 & 0.5 & 1.03 & 5809 & 4.402 & 0.035 \\
\hline HD 208704 & $1.05_{-0.03}^{+0.05}$ & $1.08_{-0.03}^{+0.05}$ & 6.9 & 0.4 & 0.98 & 5828 & 4.346 & -0.091 \\
\hline HD 210918* & $0.67_{-0.17}^{+0.06}$ & $0.71_{-0.17}^{+0.06}$ & 8.5 & 0.4 & 0.96 & 5748 & 4.319 & -0.095 \\
\hline HD $220507^{*}$ & $<0.50$ & $<0.55$ & 8.8 & 0.4 & 1.01 & 5690 & 4.247 & 0.013 \\
\hline HD $222582^{c}$ & $0.89_{-0.05}^{+0.08}$ & $0.93_{-0.05}^{+0.08}$ & 6.5 & 0.4 & 1.01 & 5784 & 4.361 & -0.004 \\
\hline Sun & $1.03_{-0.02}^{+0.03}$ & $1.07_{-0.02}^{+0.03}$ & 4.6 & - & 1.00 & 5777 & 4.438 & 0.000 \\
\hline
\end{tabular}

Notes. ${ }^{(*)} \alpha$-enhanced star. ${ }^{\left({ }^{a}\right)}$ Detected planet with $1.8 M_{\text {Jup }}$ (Jones et al. 2006). ${ }^{(b)}$ Detected planet with $0.04 M_{\text {Jup }}$ (Mayor et al. 2011). ${ }^{(c)}$ Detected planet with $7.8 M_{\text {Jup }}$ (Butler et al. 2006).

combination of spectra available in the ESO Science Archive. Furthermore, a solar flux HARPS spectrum observed in reflected sunlight from the asteroid Vesta is available. This spectrum has $S / N \sim 1200$ and was used in a differential analysis of the stars relative to the Sun.

Details about how the HARPS spectra were normalized and used to determine stellar parameters and abundances by a model atmosphere analysis of equivalent widths are given in Nissen (2015). Here we just mention that $T_{\text {eff }}$ and $\log g$ were determined by requesting that the iron abundances derived have no systematic dependence on excitation and ionization potential of the lines applied. The estimated errors of the parameters are $\sigma\left(T_{\text {eff }}\right)= \pm 6 \mathrm{~K}$ and $\sigma(\log g)= \pm 0.012$ dex, and abundances were determined with a typical precision of \pm 0.01 dex. Such extremely high precision is obtainable when high-resolution spectra of solar twin stars with $S / N>500$ are analyzed strictly differentially (line-by-line) relative to a spectrum of sunlight reflected from an asteroid observed with the same spectrograph as the stars (Bedell et al. 2014).

As seen from Table 1, the solar twin stars range from 5690 to $5870 \mathrm{~K}$ in $T_{\text {eff }}, 4.25$ to $4.50 \mathrm{dex}$ in $\log g$, and -0.11 to $+0.11 \mathrm{dex}$ in $[\mathrm{Fe} / \mathrm{H}]$. Hence, they lie in a region of the $T_{\text {eff }}-\log g$ diagram, where isochrones are not well separated (see Fig. 5 in Nissen 2015). Still, the small errors of $T_{\text {eff }}$ and $\log g$ make it possible to derive precise ages by comparing with the Yonsei-Yale set of isochrones (Yi et al. 2001; Kim et al. 2002). These ages are given in Table 1 together with stellar masses estimated from the evolutionary tracks of Yi et al. (2003). A precision of $\pm 0.01 M_{\odot}$ is estimated for the masses.

As seen from Table 1, the errors of the ages range from 0.4 to 0.8 Gyr. A comparison with ages derived by Ramírez et al. (2014) from independent high $S / N$ spectra of 14 of the stars shows good agreement within these estimated errors (see Fig. 6 in Nissen 2015). Furthermore, the abundance ratio [Y/Mg] shows a tight correlation with stellar age in a way that can be explained from the nucleosynthesis of these elements, i.e., delayed production of yttrium in low-mass AGB stars and prompt formation of magnesium in high mass, core collapse supernovae (Nissen 2015). This correlation supports that the estimated age errors are realistic in a relative sense; the absolute ages for the youngest and the oldest stars could be more uncertain.

\section{Analysis}

An inspection of the observed spectra (Fig. 1) shows that there is a clear weakening of the Li feature with increasing ages.

In order to obtain the lithium abundances, first we had to estimate the macroturbulence and rotational velocity broadening. The macroturbulence velocity was determined by the equation $v_{\text {macro }, \star}=v_{\text {macro }, \odot}+\left(T_{\text {eff }}-5777\right) / 486 \mathrm{~km} \mathrm{~s}^{-1}$ as given in 
M. Carlos et al.: Correlation between lithium abundances and ages of solar twin stars

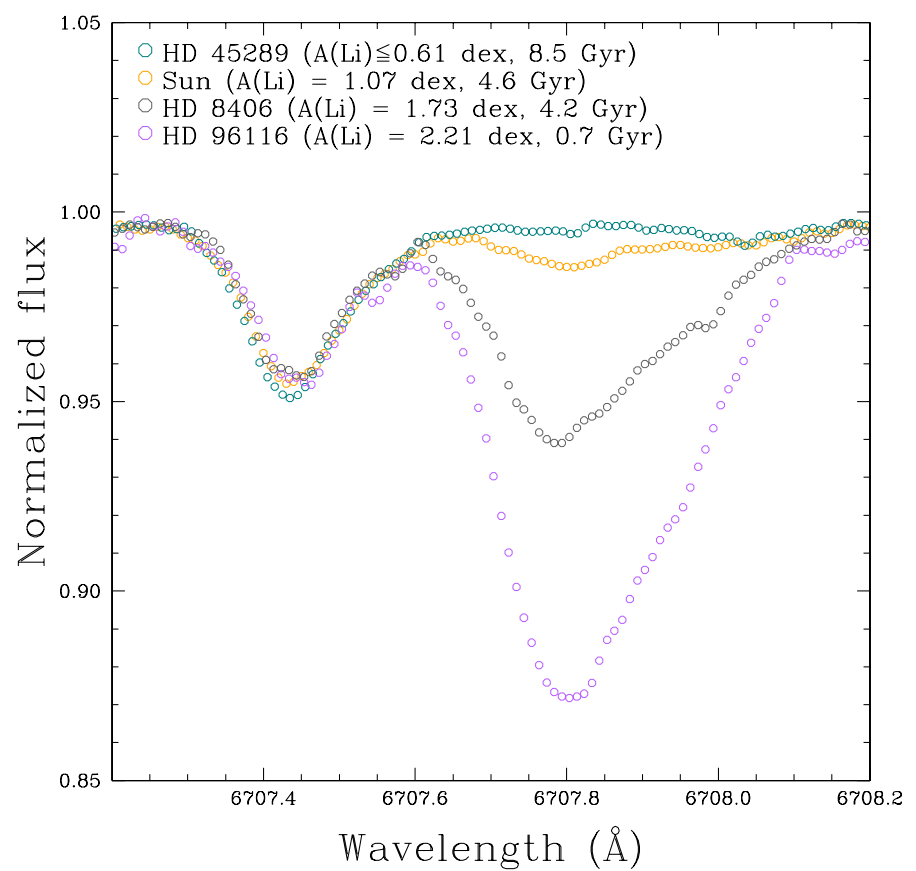

Fig. 1. Observed spectra for four stars with different ages. Notice the weakening of the Li feature for increasing ages.

Tucci Maia et al. (2015). From our analysis of the HARPS solar spectrum we estimated $v_{\text {macro, } \odot}=3.2 \mathrm{~km} \mathrm{~s}^{-1}$, adopting $v \sin i_{\odot}=$ $1.9 \mathrm{~km} \mathrm{~s}^{-1}$. The projected rotational velocity was calculated by analyzing the line profiles of the Fe I $6027.050 \AA$, $6093.644 \AA$, $6151.618 \AA, 6165.360 \AA, 6705.102 \AA$, and Ni I $6767.772 \AA$ lines. Notice that the instrumental broadening was also included.

The lithium abundances were estimated by spectral synthesis in the region of the $6707.75 \AA$ Li I line using the July 2014 version of the 1D LTE code MOOG (Sneden 1973) and the Kurucz new grid of ATLAS9 model atmospheres (Castelli \& Kurucz 2004). The line list adopted for the Li region is from Meléndez et al. (2012), and includes blends from atomic and molecular $\left(\mathrm{CN}\right.$ and $\left.\mathrm{C}_{2}\right)$ lines; the dependence of the different species is shown in Fig. 2. An example of spectral synthesis for HD 45184 is shown in Fig. 3, where it can be seen that the Li line profile changes very significantly for an abundance difference of 0.1 dex.

The abundances were derived assuming LTE and then NLTE results were obtained with the aid of the INSPECT database ${ }^{1}$, based on NLTE calculations by Lind et al. (2009). The errors were obtained taking into account uncertainties in the continuum setting, the rms deviation of the observed line profile relative to the synthetic profile, and the stellar parameters. Both LTE and NLTE values of $A(\mathrm{Li})=\log \left(N_{\mathrm{Li}} / N_{\mathrm{H}}\right)+12$ with their respective errors are shown in Table 1.

It is important to note that our results in LTE compare well with the large study by Delgado Mena et al. (2014), as shown in Fig. 4. Compared to previous works on solar twins (Monroe et al. (2013) for 18 Sco and HIP 102152, Meléndez et al. (2012) for HIP 56948, Meléndez et al. (2014) for HIP $114328^{2}$ and

\footnotetext{
1 WWW.inspect-stars. com (version 1.0).

2 A new better spectrum of HIP 114328 has been recently acquired by one of the authors (J.M.). The stellar parameters are $T_{\text {eff }}=5763 \pm 12 \mathrm{~K}$, $\log g=4.33 \pm 0.04 \mathrm{dex}$ and $[\mathrm{Fe} / \mathrm{H}]=-0.050 \pm 0.014 \mathrm{dex}$, resulting in an age of $7.6_{-0.9}^{+0.6} \mathrm{Gyr}$ and mass equal to $1.00 \pm 0.01 M_{\odot}$. Considering these new results the star fits better now the Li-age trend.
}

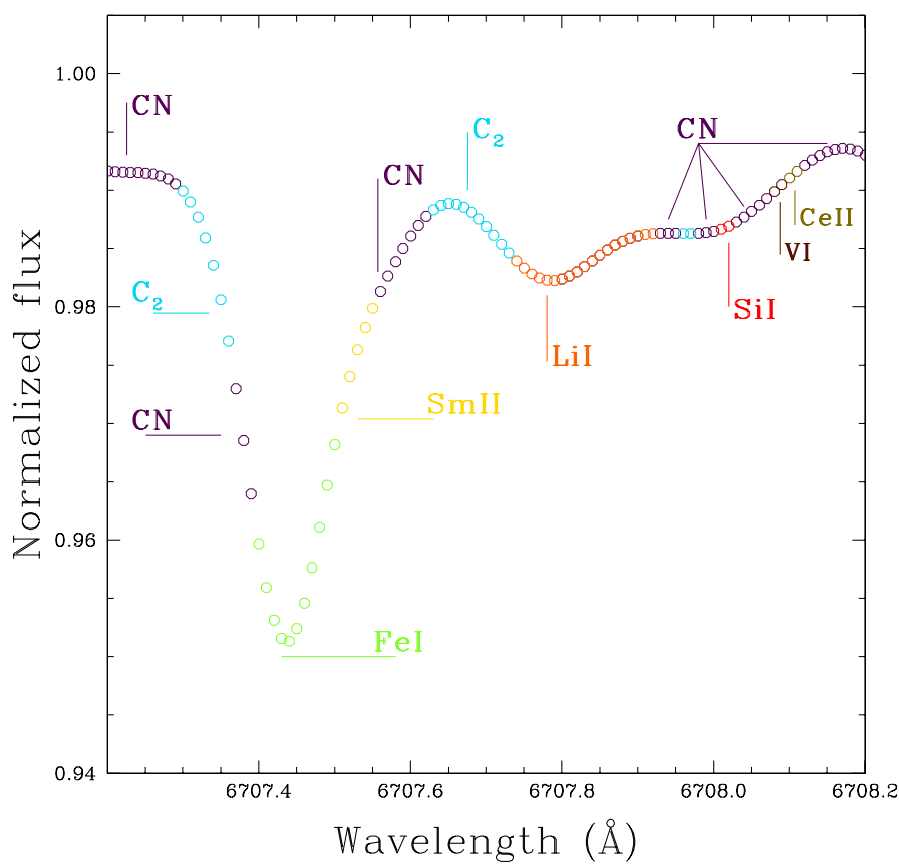

Fig. 2. Synthetic solar spectrum considering different species in the region of the $6707.75 \AA \mathrm{Li}$ I line.

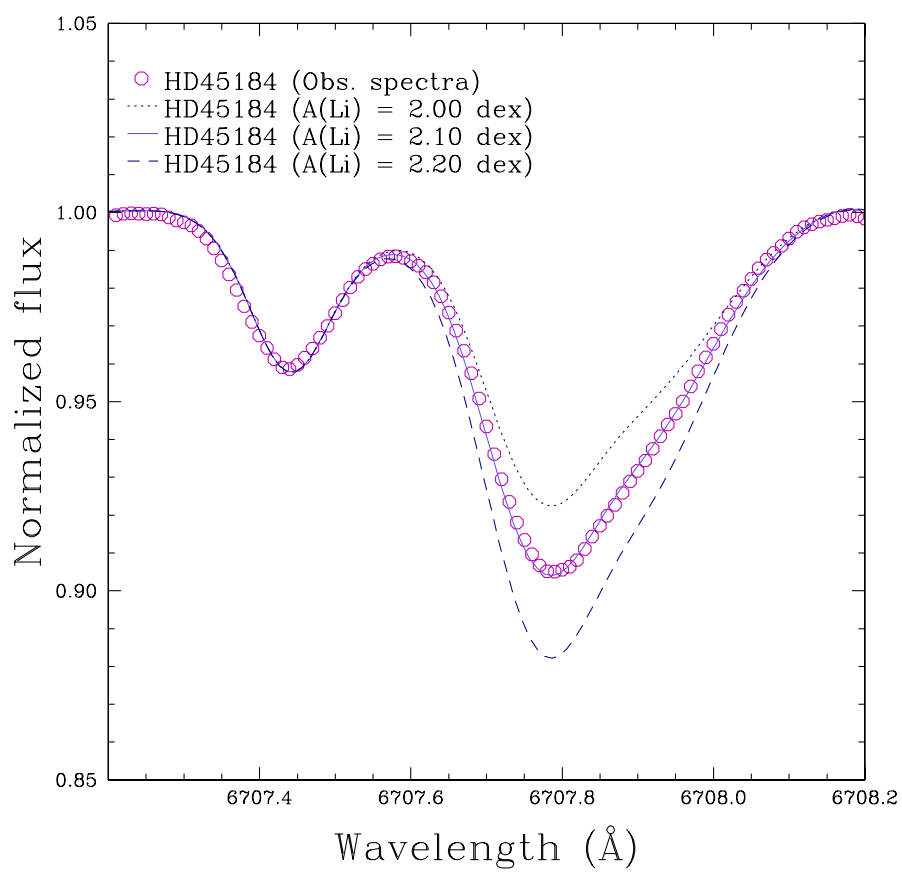

Fig. 3. Open circles represent the observed spectra for HD 45184 in comparison with three different synthetic spectra.

Ramírez et al. (2011) for 16 Cyg A and 16 Cyg B), our results, now in NLTE, follow the same lithium age trend, as illustrated in Fig. 5. The effective temperatures and surface gravities of the stars in these papers were determined in the same way as in Nissen (2015), i.e., by a differential analysis of Fe lines relative to the Sun, and ages were also obtained by interpolating between Yonsei-Yale isochrones. Hence, we expect that the $\mathrm{Li}$ abundances and ages are on the same system as in the present paper. 


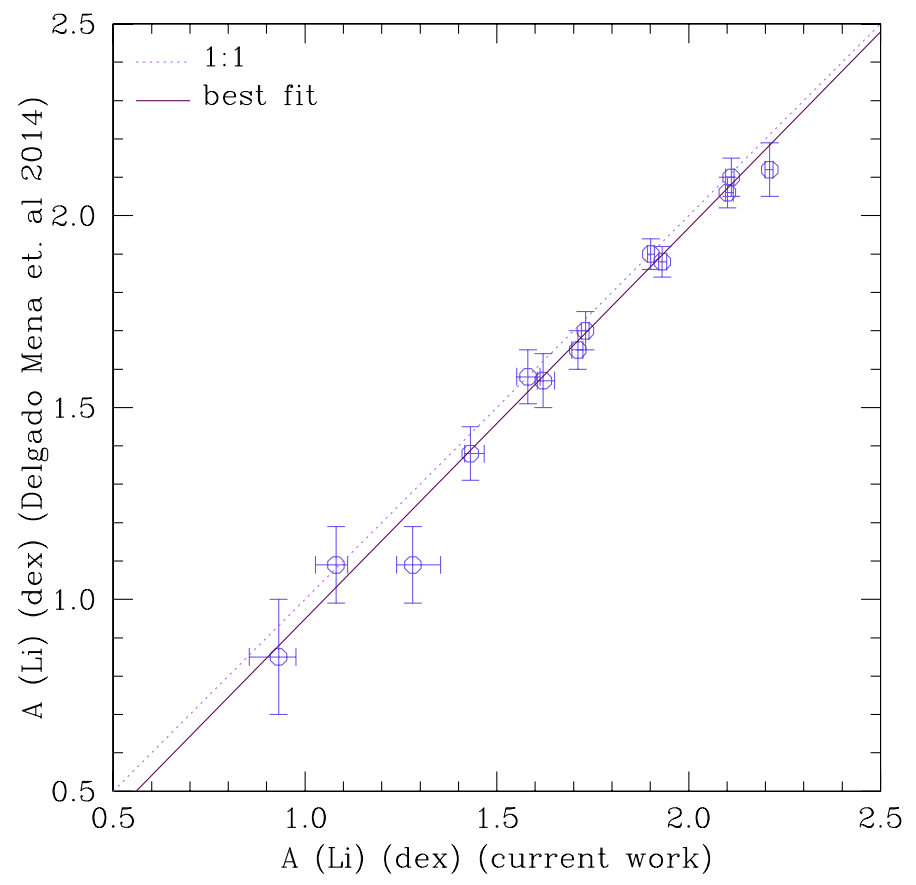

Fig. 4. LTE Li abundances for our sample compared with LTE data from the work of Delgado Mena et al. (2014).

\section{Discussion}

The results for the whole sample can be seen in Fig. 5, where we notice a strong connection between lithium abundance and stellar age. 16 Cyg A and two stars in our sample, namely HD 96423 and HD 38277, have higher abundances than expected in comparison to both models and the other stars. A possible explanation for this $\mathrm{Li}$ enhancement could be planet engulfment as described in Montalbán \& Rebolo (2002) and Sandquist et al. (2002).

Another interesting result is that the $\alpha$-enhanced stars (represented by teal blue filled circles in Fig. 5) fall on the same Li-age sequence as the thin disk solar twins. Two of these $\alpha$-enhanced stars (HD 45289 and HD 210918) have thick-disk kinematics, while the third one (HD 220507) has thin-disk kinematics like the rest of the stars (Nissen 2015), but this latter star has a chemical abundance pattern consistent with the other two thick disk stars.

Considering all stars in our sample for which a $\mathrm{Li}$ abundance has been derived (i.e., excluding stars with only an upper limit), the Li-age correlation has about a $10 \sigma$ significance; we performed a Spearman test and found a Spearman rank coefficient $r_{\mathrm{s}}=-0.89$ and a probability of $10^{-8}$ of our results arising by pure chance. Therefore, even when the two deviant stars are considered the correlation between Li and age is very strong.

Excluding the anomalous stars HD 96423 and HD 38277, the Li-age correlation for 17 stars in our sample that have firm detections of the $\mathrm{Li}$ line is at the $23 \sigma$ significance level and has a very small probability of not being real $\left(10^{-11}\right)$ with a Spearman rank coefficient $r_{\mathrm{s}}=-0.96$, meaning an almost perfect Spearman correlation. This is in contrast to the poor correlation found by Delgado Mena et al. (2014; their Fig. 8). Notice, however, that they include stars with masses lower and higher than the Sun's and they also include stars in a broader metallicity range. The wider interval in both metallicity and mass apparently blurs the strong correlation that we find.

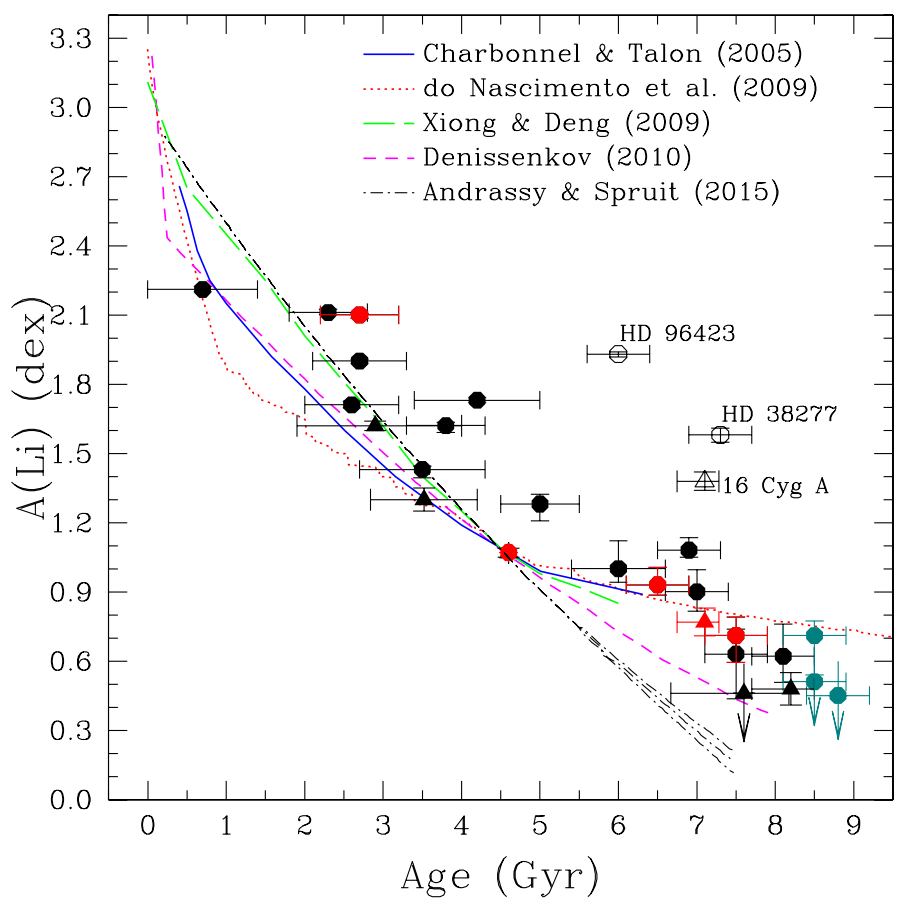

Fig. 5. Connection between stellar ages and NLTE lithium abundances for our current sample (circles) and some previous results (triangles) referenced in the text. Teal blue circles indicate alpha-enhanced stars and red symbols refer to stars hosting planets. The models of Li depletion were normalized to the solar $\mathrm{Li}$ abundance. In some cases, the lithium abundance errors are smaller than the points.

In Fig. 5 we compare our Li-age results with Li depletion models for a one solar mass star with solar metallicity (Xiong \& Deng 2009; Do Nascimento et al. 2009; Charbonnel \& Talon 2005; Denissenkov 2010; Andrássy \& Spruit 2015) that take into account physics not included in the standard solar model. Different transport mechanisms are explored in these models, including meridional circulation, diffusion (gravitational settling and radiative acceleration), turbulence, gravity waves, convective overshooting, and convective settling. Overall, all models have a reasonable qualitative agreement with the data, as expected because they were calibrated using the Sun or earlier observations of solar twins. With the data presented here, the models above could be improved. However, such refinements should take into account that the initial Li abundance of stars probably have increased in time due to Galactic evolution, i.e., Li production in AGB stars and novae (Chen et al. 2001; Romano et al. 2001; Lambert \& Reddy 2004; Ramírez et al. 2012). The models assume an initial $\mathrm{Li}$ abundance of $A(\mathrm{Li})=$ 3.3 as in meteorites, but according to the chemical evolution model of Izzo et al. (2015), the initial Li abundance has increased from about $A(\mathrm{Li})=2.8$ for the oldest disk stars to about $A(\mathrm{Li})=$ 3.6 for the youngest. Taking this into account would make the $A(\mathrm{Li})$ - age relation predicted by the models steeper than shown in Fig. 5.

For models including rotational induced mixing, variations of the initial rotational velocity cause a considerable scatter in $A(\mathrm{Li})$ at a given age; according to Charbonnel \& Talon (2005) a

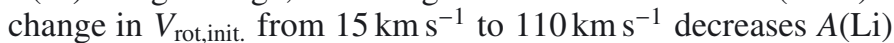
by about 1 dex for the oldest stars. Differences in the initial rotational velocity may, therefore, contribute to the scatter seen in Fig. 5, although very large differences in $V_{\text {rot,init. }}$ are needed to explain the outliers. 

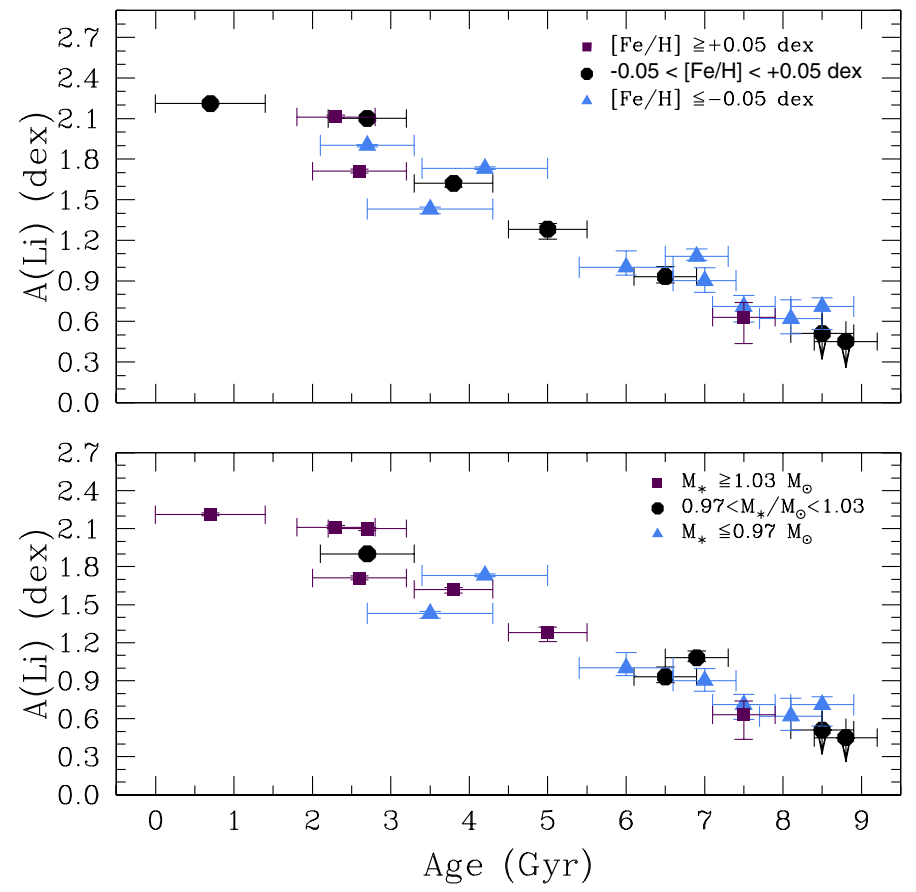

Fig. 6. Connection between stellar ages and lithium abundances with different symbols for three metallicity groups (top) and three mass groups (bottom).

An alternative explanation for enhanced lithium depletion is the presence of planets. Israelian et al. (2009) suggest that planet hosting stars have less lithium than stars without planets, but Baumann et al. (2010) contest this result claiming that the sample of the former work is biased in metallicity and includes stars of different evolutionary stages. In agreement with Baumann et al. (2010), Fig. 5 shows a lack of connection between lithium depletion and presence of planets. There is no significant segregation between stars without planets (black filled symbols) and planet hosting stars (red filled symbols) in this figure.

As shown in Fig. 6, where the stars in our sample have been divided into three metallicity groups (upper panel) and three mass groups (lower panel), there is no obvious indication of a dependence of lithium depletion on metallicity and mass at a given age. An unweighted linear least squares fit to $A(\mathrm{Li})$ as a function of age (in Gyr) for stars with a detected Li line (including the Sun) leads to

$A(\mathrm{Li})=2.437( \pm 0.098)-0.224( \pm 0.018)$ Age.

Fitting the residuals of $A(\mathrm{Li})$ relative to this equation as a function of $[\mathrm{Fe} / \mathrm{H}]$ and mass results in the relation

$$
\begin{aligned}
\Delta A(\mathrm{Li})= & -0.05-2.17( \pm 1.30)[\mathrm{Fe} / \mathrm{H}] \\
& +3.55( \pm 2.25)\left(M / M_{\odot}-1.0\right) .
\end{aligned}
$$

Although, the coefficients have the expected sign, i.e., increasing Li depletion with increasing metallicity and decreasing mass (e.g., Castro et al. 2009), the dependence is hardly significant, i.e., a $1.7 \sigma$ correlation with metallicity and a $1.6 \sigma$ correlation with mass. Furthermore, the mass dependence is smaller than predicted from models (Xiong \& Deng 2009; Castro et al. 2009), and also smaller than inferred from Li abundance measurements for stars in the open cluster M67, which suggests a mass coefficient on the order of 10 for $\sim 1 M_{\odot}$ stars (Pace et al. 2012). Apparently, the short mass range for our sample (from $0.94 M_{\odot}$ to $1.07 M_{\odot}$ ) in combination with errors in age, mass, and $\mathrm{Li}$ abundance make it difficult to estimate the dependence of Li abundance on mass based on our sample. We note, however, that according to our results, it is unlikely that the large difference in $A(\mathrm{Li})$ between $16 \mathrm{Cyg} \mathrm{A}$ and $16 \mathrm{Cyg} \mathrm{B}$ is due to the difference in mass $\left(0.05 M_{\odot}\right)$ as suggested by Ramírez et al. (2011). Instead, the high Li abundance in 16 Cyg A may be due to a recent accretion of a Jupiter sized planet, which is consistent with the fact that no such planet has been detected for $16 \mathrm{Cyg} \mathrm{A}$, whereas 16 Cyg B has a giant planet with a minimum mass of $1.5 M_{\text {Jup }}$ (Cochran et al. 1997). A related evidence supporting the planet engulfment scenario could be the enhanced abundances for all elements in 16 Cyg A relative to 16 Cyg B (Ramírez et al. 2011; Tucci Maia et al. 2014), and in particular for the refratory elements (Tucci Maia et al. 2014).

\section{Conclusions}

Based on HARPS spectra with very high signal-to-noise $(S / N \geq$ $600)$ we were able to determine high-precision lithium abundances for 21 solar twin stars for which isochrone ages have been estimated from the $T_{\text {eff }}-\log g$ diagram. Excluding two outliers, we see a strong correlation between stellar ages and lithium abundances, with a $23 \sigma$ significance and a Spearman test coefficient of $r_{\mathrm{s}}=-0.96$. In addition, five solar twins for which high-precision lithium abundances and ages were previously derived (Monroe et al. 2013; Meléndez et al. 2012, 2014; Ramírez et al. 2011) and the Sun itself fit the relation very well. Over an age range from 1 to $9 \mathrm{Gyr}$, the $\mathrm{Li}$ abundance decreases by almost 2 dex, which we interpret as being due to gradual destruction of lithium near the bottom of the outer convection zone. Thus, the new data can be used to constrain non-standard models of Li depletion, and therefore to understand better the transport mechanisms inside stars.

Two stars deviate from the main trend between $A(\mathrm{Li})$ and age by having $\mathrm{Li}$ abundances enhanced by a factor of $\sim 10$. A similar enhancement of $\mathrm{Li}$ is present for $16 \mathrm{Cyg} \mathrm{A}$ relative to $16 \mathrm{Cyg} \mathrm{B}$ even though the two stars have the same age and nearly the same mass and metallicity. We suggest that these enhancements of $\mathrm{Li}$ are connected to the accretion of Jupiter sized planets.

We find no evidence for a link between planet hosting stars and enhanced Li destruction as suggested in some recent works. Three stars with planets of different masses in our sample, 16 Cyg B with a Jupiter sized planet (Ramírez et al. 2011), and the Sun show no systematic deviation from the $A(\mathrm{Li})$-age relation for stars without planets.

Acknowledgements. The referee is thanked for important comments, that helped to improve this paper. M.C. would like to acknowledge support from CAPES. P.E.N. acknowledges support from the Stellar Astrophysics Centre funded by The Danish National Research Foundation (Grant agreement No.: DNRF106) and from the ASTERISK project (ASTERoseismic Investigations with SONG and Kepler) funded by the European Research Council (Grant agreement No.: 267864). J.M. would like to acknowledge support from FAPESP (2012/24392-2) and CNPq (Bolsa de Produtividade).

\section{References}

Andrássy, R., \& Spruit, H. C. 2015, A\&A, 579, A122

Baumann, P., Ramírez, I., Meléndez, J., Asplund, M., \& Lind, K. 2010, A\&A, 519, A87

Bedell, M., Meléndez, J., Bean, J. L., et al. 2014, ApJ, 795, 23

Butler, R. P., Wright, J. T., Marcy, G. W., et al. 2006, ApJ, 646, 505

Castelli, F., \& Kurucz, R. L. 2004, ArXiv e-prints [arXiv:astro-ph/0405087]

Castro, M., Vauclair, S., Richard, O., \& Santos, N. C. 2009, A\&A, 494, 663

Charbonnel, C., \& Talon, S. 2005, Science, 309, 2189

Chen, Y. Q., Nissen, P. E., Benoni, T., \& Zhao, G. 2001, A\&A, 371, 943

Cochran, W. D., Hatzes, A. P., Butler, R. P., \& Marcy, G. W. 1997, ApJ, 483, 457 
Delgado Mena, E., Israelian, G., González Hernández, J. I., et al. 2014, A\&A, 562, A92

Denissenkov, P. A. 2010, ApJ, 719, 28

Do Nascimento, Jr., J. D., Castro, M., Meléndez, J., et al. 2009, A\&A, 501, 687

Figueira, P., Faria, J. P., Delgado-Mena, E., et al. 2014, A\&A, 570, A21

Gonzalez, G. 2015, MNRAS, 446, 1020

Gonzalez, G., Carlson, M. K., \& Tobin, R. W. 2010, MNRAS, 403, 1368

Israelian, G., Delgado Mena, E., Santos, N. C., et al. 2009, Nature, 462, 189

Izzo, L., Della Valle, M., Mason, E., et al. 2015, ApJ, 808, L14

Jones, H. R. A., Butler, R. P., Tinney, C. G., et al. 2006, MNRAS, 369, 249

Kim, Y.-C., Demarque, P., Yi, S. K., \& Alexander, D. R. 2002, ApJS, 143, 499

Lambert, D. L., \& Reddy, B. E. 2004, MNRAS, 349, 757

Lind, K., Asplund, M., \& Barklem, P. S. 2009, A\&A, 503, 541

Mayor, M., Pepe, F., Queloz, D., et al. 2003, The Messenger, 114, 20

Mayor, M., Marmier, M., Lovis, C., et al. 2011, A\&A, submitted [arXiv: 1109.2497]

Meléndez, J., Bergemann, M., Cohen, J. G., et al. 2012, A\&A, 543, A29

Meléndez, J., Schirbel, L., Monroe, T. R., et al. 2014, A\&A, 567, L3

Monroe, T. R., Meléndez, J., Ramírez, I., et al. 2013, ApJ, 774, L32

Montalbán, J., \& Rebolo, R. 2002, A\&A, 386, 1039
Nissen, P. E. 2015, A\&A, 579, A52

Pace, G., Castro, M., Meléndez, J., Théado, S., \& do Nascimento, Jr., J.-D. 2012, A\&A, 541, A150

Ramírez, I., Meléndez, J., Cornejo, D., Roederer, I. U., \& Fish, J. R. 2011, ApJ, 740,76

Ramírez, I., Fish, J. R., Lambert, D. L., \& Allende Prieto, C. 2012, ApJ, 756, 46 Ramírez, I., Meléndez, J., Bean, J., et al. 2014, A\&A, 572, A48

Romano, D., Matteucci, F., Ventura, P., \& D’Antona, F. 2001, A\&A, 374, 646

Sandquist, E. L., Dokter, J. J., Lin, D. N. C., \& Mardling, R. A. 2002, ApJ, 572, 1012

Sneden, C. A. 1973, Ph.D. Thesis, The University of Texas at Austin

Sousa, S. G., Santos, N. C., Mayor, M., et al. 2008, A\&A, 487, 373

Sousa, S. G., Fernandes, J., Israelian, G., \& Santos, N. C. 2010, A\&A, 512, L5

Takeda, Y., Honda, S., Kawanomoto, S., Ando, H., \& Sakurai, T. 2010, A\&A, 515, A93

Tucci Maia, M., Meléndez, J., \& Ramírez, I. 2014, ApJ, 790, L25

Tucci Maia, M., Meléndez, J., Castro, M., et al. 2015, A\&A, 576, L10

Xiong, D. R., \& Deng, L. 2009, MNRAS, 395, 2013

Yi, S., Demarque, P., Kim, Y.-C., et al. 2001, ApJS, 136, 417

Yi, S. K., Kim, Y.-C., \& Demarque, P. 2003, ApJS, 144, 259 